\title{
Five-year institutional experience on the impact of afirma and thyroseq $v 2$ on indeterminate thyroid nodules for malignancy prediction
}

\begin{abstract}
Design: In a retrospective study at Queens Hospital Center, medical records of 1010 patients with thyroid nodule over a five year period (Jan 2012-Dec 2016) was studied and rates of malignancy, molecular marker test (MMT) results, and number of cases of averted thyroidectomy were calculated.

Results: Total of 151 patients were found to have indeterminate FNA cytology results; 85 patients had no MMTs, 38 had results from Thyroseqv2, and 28 had results from Afirma. Out of 85 patients with no MMT, 42 patients (49\%) had a thyroidectomy which $9(21 \%)$ had thyroid cancer and 33(79\%) had benign pathology. Seven patients with a positive Thyroseqv2 mutational panel had a thyroidectomy and of these, $5(71 \%)$ were found malignant. 3 patients with negative Thyroseqv2 had a thyroidectomy due to nodule growth, and one had malignancy. With the use of Thyroseqv2, 22 patients out of $38(57 \%)$ were spared unnecessary thyroidectomy. Of 28 patients who had Afirma results, 16(57\%) had a suspicious gene expression classifier (GEC). Among 16 patients with a suspicious GEC, 11 had a thyroidectomy and $4(36 \%)$ had cancer. GEC testing was able to spare 12 patients $(42 \%)$ from thyroidectomy.

Conclusions: Using MMTs, 34(51\%) patients out of 66 were spared unnecessary thyroidectomy. Due to a rather small sample size, statistical accuracy of MMTs has not been reported. Based on review of the literature, MMTs are very helpful in further analyzing indeterminate nodules and prevents unnecessary surgery given negative (both Thyroseqv2 and Afirma) and positive (Thyroseqv2) predictive values.
\end{abstract}

Keywords: thyroid nodule, indeterminate Bethesda, molecular testing, thyroSeq V2, afirma
Volume 5 Issue 6 - 2017

\section{Sara Shapouran,' Seyedmohammad Pourshahid, ${ }^{2}$ Maria Fuzailov, ${ }^{3}$ Issac Sachmechi ${ }^{4}$}

'Research volunteer at Endocrinology Division, Department of Medicine, Icahn School of Medicine at Mount Sinai/NYC Health+Hospitals/ Queens, USA

Internal Medicine resident at Department of Medicine, Icahn School of Medicine at Mount Sinai/NYC Health+Hospitals/ Queens, USA

${ }^{3}$ Medical student at Department of Medicine, Icahn School of Medicine at Mount Sinai/NYC Health+Hospitals/Queens, USA ${ }^{4}$ Associate Professor of Endocrinology, Department of Medicine, Icahn School of Medicine at Mount Sinai/NYC Health+Hospitals/Queens, USA

Correspondence: Seyedmohammad Pourshahid, Resident physician in Internal medicine program of Mount Sinai at Queens Hospital Center, 82-68 164th Street, Department of internal medicine at Queens hospital Center, Jamaica, NY I I 432, USA, Tel + I (60I) 20I-5I68,

Email Seyedmohammad.Pourshahid@mssm.edu,Pourshas@ nychhc.org

Received: July 24, 2017 | Published: August |4, 2017
Abbreviations: BSRTC, bethesda system for reporting thyroid cytopathology; ACUS, atypia of undetermined significance; FLUS, follicular lesion of undetermined significance; SFN, suspicious for follicular neoplasm; MMTs, molecular marker tests; GEC, gene expression classifier

\section{Introduction}

Estimated thyroid nodule prevalence found by palpation is $4-7 \%$ 1 and it may be found in up to 20-76 of population by ultrasound. ${ }^{1,2}$ It is usually the first sign being noticed for thyroid cancer. For the initial assessment Fine needle aspiration has been widely used as a fast, cost effective and reliable way of further evaluation which usually separating the group of patient $\mathrm{s}$ who will require medical versus surgical management. ${ }^{3}$ Thyroid cancer has the highest growth of incidence in United States. In projection of cancer incidence of 2020 to 2030 Will replace colorectal by year 2030 as the fourth leading cancer breast, prostate and lung cancer remained the top 3 types of cancer however thyroid cancer replaced colorectal cancer in the fourth top cancer diagnosis of $2030 .{ }^{4}$ Accurate distinction of benign and malignant nodule is crucial to treat the malignancy and avoid unnecessary procedure and possible complications. The next step after FNA is determined by malignancy risk which varies in-between continuum of six groups in Bethesda System for Reporting Thyroid Cytopathology (BSRTC). ${ }^{5}$ The risk ranges from $3 \%$ in the group with benign pathology vs $99 \%$ in the ones with malignant cytopathology diagnosis. The indeterminate cytology which includes Bethesda group 3, Atypia of undetermined significance (ACUS) or Follicular lesion of undetermined significance (FLUS), and group 4, Suspicious for Follicular Neoplasm (SFN), is a gray zone and unfortunately there is high uncertainty in diagnosis. ${ }^{6} 20-30 \%$ of FNA cytology yields indeterminate diagnosis. ${ }^{5}$ Having malignancy risk of $15-30 \%$ the chance of malignancy is neither too low to defer further evaluation nor too high to indicate cancer surgery.

Current guidelines recommend repeat FNA and diagnostic lobectomy for group 3 and 4 respectively. ${ }^{8}$ Several molecular marker tests (MMTs) such as Thyroseqv2 or Afirma are used to assist determining if a nodule is benign or malignant. Initially MMTs were classified as rule in and rule out groups. ${ }^{9}$ Afirma Gene Expression Classifier(GEC) is a test based on microarray technology used to analyze the mRNA expression of 167 different genes that is based on the gene expression identified from FNAs of surgically proven benign and malignant thyroid nodules and was validated in double blinded studies. ${ }^{10}$ With Negative predictive value of $(92-95 \%)$ and positive predictive value of $37-38 \%$, Afirma is mainly considered as "rule out" test. ${ }^{11}$ ThyroSeq v2 is the enhanced version of ThyroSeq that was introduced in 2014 which can correctly detects 12 cancer genes with 284 mutational hot spots previously found positive in thyroid tumor samples and cell lines. ${ }^{11}$ ThyroSeq V2 accurately detect both 
malignant and benign samples. With positive predictive value of $83 \%$ and negative predictive value of $96 \%$, ThyroSeq V2 is used as Rule in test as well as Rule out study for indeterminate thyroid nodule. ${ }^{12,13}$

\section{Materials and methods}

A retrospective study on 1010 patients with thyroid nodule FNA results was performed at Queens Hospital Center over a five year period (Jan 2012-Dec 2016) using an electronic medical record database. Total of 151 patients with indeterminate thyroid FNA cytology results were identified. Result of MMTs and surgery were analyzed whenever available. Rates of malignancy, MMT results, and number of cases of averted thyroidectomy were calculated.

\section{Results}

The records were retrospectively reviewed of a total of 151 patients with indeterminate thyroid FNA cytology results (Figure 1). The basic characteristics of all patients in 3 groups of no MMTs, Afirma, and ThyroSeq V2 are shown in Table 1. The demographics of the data were typical for thyroid malignancy. Most of the patients were woman in all the groups. Out of 85 patients without MMTs, 42 patients had surgery. Before introduction of MMTs usually all the patients with indeterminate thyroid FNA results were recommended to have diagnostic thyroidectomy to rule out malignancy. But almost half of our patients (43) did not have surgery due to various causes. Some of the patients had scheduled for surgery out of the time-frame of our study. At the final histopathology, 9/42 nodules were malignant and 33/42 were benign. All of the malignancies were papillary carcinoma of thyroid (PCT). 33 patients who had thyroidectomy with the final benign histopathology are the patients who could potentially benefit from MMTs, assuming the accuracy of the MMTs are very high.

MMTs were performed on 66 indeterminate nodules, 38 ThyroSeq V2 and 28 Afirma. In Afirma group, 16/28 patients had suspicious gene expresser classifier results. Among 16 patients with a suspicious GEC, 11 had a thyroidectomy and 4(36\%) had cancer. All 4 cancerous results were PCT. One of the patients with a benign GEC result had surgery due to the size of the nodule which the result of final histopathology was also benign, confirming the result of GEC. 11 patients did not have diagnostic thyroidectomy counting on the benign result of GEC. Of the 38 patients who had Thyroseqv2 results, 13(42\%) had a positive mutational panel, with 12 harboring a positive RAS mutation and 1 harboring a positive BRAF mutation. Seven patients with a positive Thyroseqv 2 mutational panel had a thyroidectomy and, of these, $5(71 \%)$ were found to be cancerous. $2 / 5$ cancers were noninvasive follicular thyroid neoplasm with papillary-like nuclear features (NIFPT) and 3/5 were PCT. 2 patients harboring NRAS and KRAS mutations on ThyroSeq V2, had final benign histopathology. On the other hand, 3 of the patients with negative mutational panels in Thyroseqv 2 had a thyroidectomy due to nodule growth, and 1of them had PCT, tall cell variant. With the use of Thyroseqv2, 22 patients out of $38(57 \%)$ were spared unnecessary thyroidectomy. The results of the nodules with MMTs are shown in Figure 2. With the use of Afirma GEC, 7 patients had false positive results, while 4 had true positive results. With the use of ThyroSeq V2, 2 patients had false positive results, while 5 had true positive results. In terms of Specificity of the tests, we had only 4 patients with MMTs who had thyroidectomy; 1 in Afirma group which was true negative and 3 in ThyroSeq V2 group, 2 were true negative and 1 false negative.

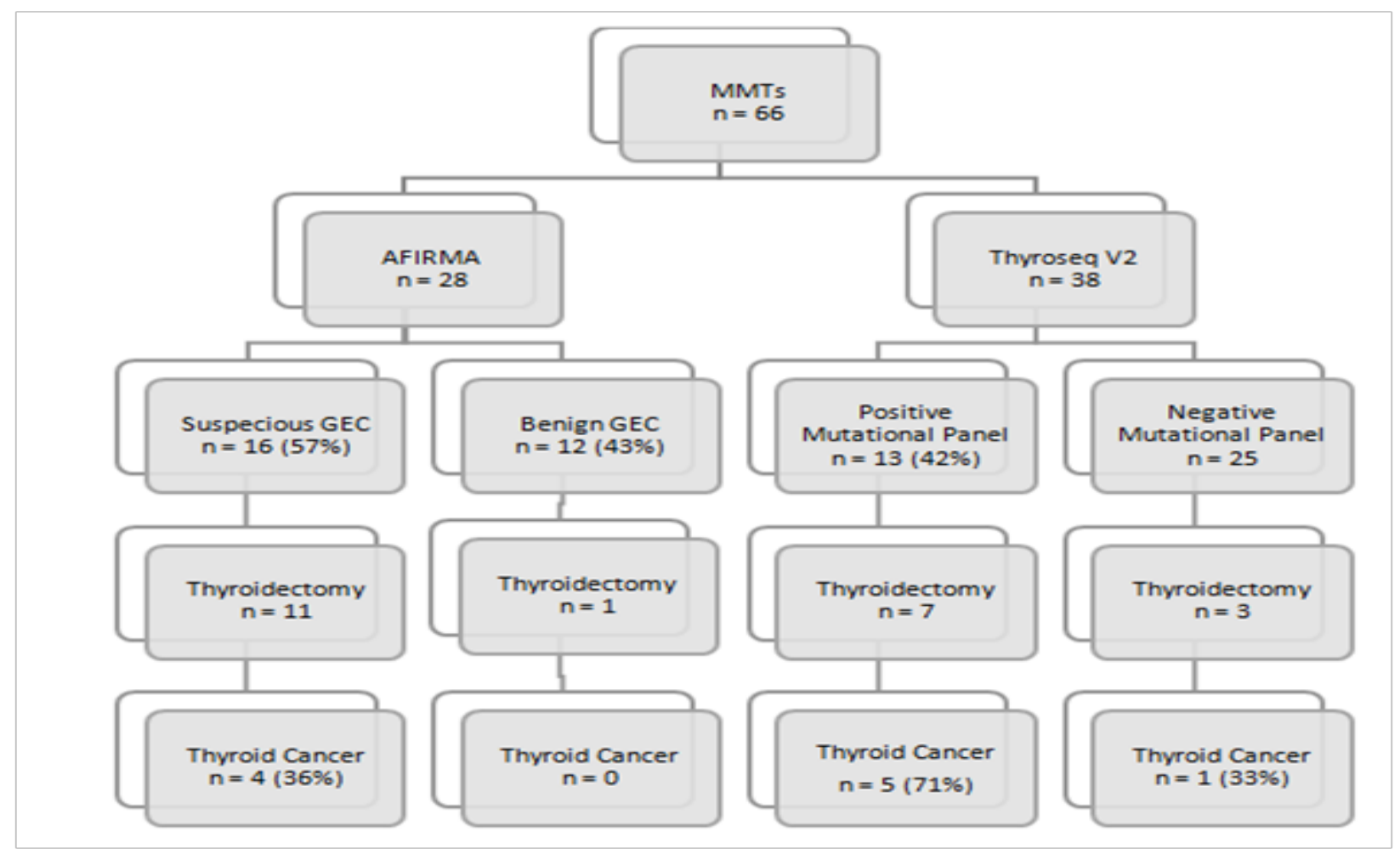

Figure I Schematic representation of the results in 66 patients with MMTs. 


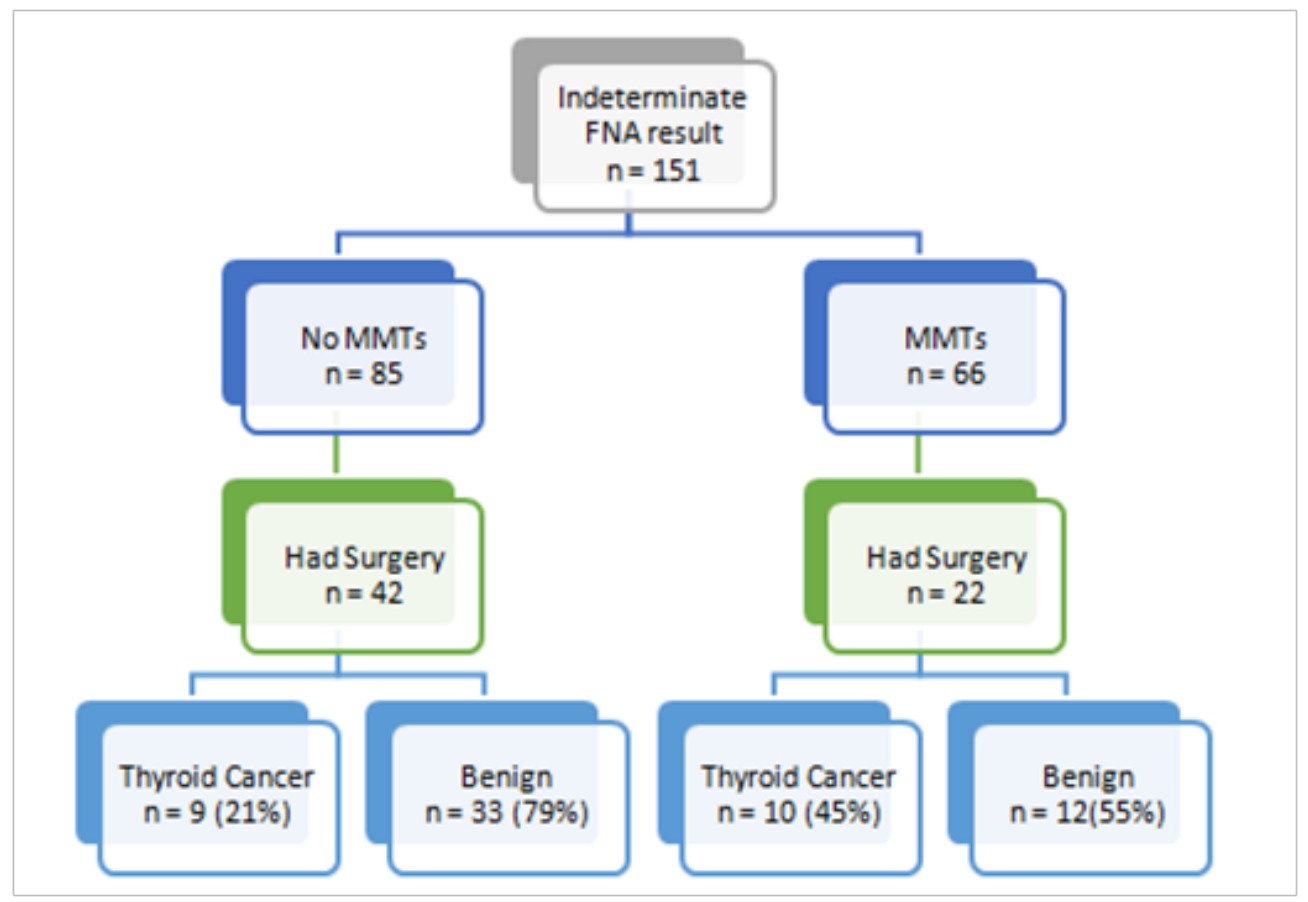

Figure 2 Schematic representation of the results in all patients with indeterminate FNA result included in the study.

Table I Demographics

\begin{tabular}{llll}
\hline & NO MMts & Afirma & Thyroseq V2 \\
\hline Total & 85 & 28 & 38 \\
Age (Mean-SD) & $56.8-13.11$ & $62.3-8.8$ & $50.5-16.4$ \\
Female gender & 76 & 25 & 29 \\
Surgery & 42 & 12 & 10 \\
\hline
\end{tabular}

\section{Discussion}

In this study, we emphasized the importance of using MMTs in sparing patients with indeterminate nodules from unnecessary thyroidectomy. With the use of MMTs, 34 (51\%) patients out of 66 were spared unnecessary thyroidectomy. The Afirma GEC test, offers a high negative predictive value (NPV) and relatively low positive predictive value (PPV) ${ }^{11}$ In our study the predicting results of Afirma test and final surgery was roughly the same as previous literature. Thyroseq V2 offers high NPV and PPV due to the ability to check many of the genetic markers and point mutations. ${ }^{11,12}$ In our study we had 5/7 true positive results which was almost the same as the promised PPV of the test. 2 patients with false positive results, had NRAS and KRAS mutations which has the lower risk of cancer in comparison to HRAS and BRAF mutation. ${ }^{12}$ One of 3 patients with negative Thyroseqv2 mutational panels had surgery due to increases in size of the nodule was found to have PCT. Due to a rather small sample size, this paper cannot comment on the true statistical accuracy of MMTs.

\section{Conclusion}

MMTs are very important in further stratification of the indeterminate thyroid nodules (Bethesda category III-IV) into benign and malignant, resolving the diagnostic uncertainty, and sparing unnecessary thyroidectomy. In this study we assessed the performance of MMTs in our institution in a period of 5 years. Based on our results, $51 \%$ of patients who had MMT results spared unnecessary thyroidectomy. However vigilant follow-up for changes in characteristics or growth of nodules to clinically validate MMTs is recommended as 1 of our patients with negative mutational panel had PCT pathology result after surgery.

\section{Acknowledgements}

None.

\section{Conflict of interest}

Authors declare no conflict of interest.

\section{References}

1. Popoveniuc G, Jonklaas J. Thyroid nodules. Med Clin North Am. 2002;96(2):329-349.

2. Mazzaferri EL. Management of a solitary thyroid nodule. The New England journal of medicine. 1993;328(8):553-559.

3. Ross DS. Editorial: predicting thyroid malignancy. J Clin Endocrinol Metab. 2006;91(11): 4253-4255.

4. Rahib L, Smith BD, Aizenberg R, et al. Projecting cancer incidence and deaths to 2030: the unexpected burden of thyroid, liver, and pancreas cancers in the United States. Cancer Res. 2014;74(11):2913-2921.

5. Shrestha RT, Evasovich MR, Amin K, et al. Correlation between histological diagnosis and mutational panel testing of thyroid nodules: a two-year institutional experience. Thyroid. 2016;26(8):1068-1076. 
6. Haugen BR, Alexander EK, Bible KC, et al. The American thyroid association guidelines task force on thyroid nodules and differentiated thyroid cancer. Thyroid. 2016;26(1):1-133.

7. Bongiovanni M, Spitale A, Faquin WC, et al. The Bethesda System for Reporting Thyroid Cytopathology: a meta-analysis. Acta Cytol. 2012;56(4):333-339.

8. Gharib H, Papini E, Garber JR, et al. American association of clinical endocrinologists, american college of endocrinology, and associazione medici endocrinologi medical guidelines for clinical practice for the diagnosis and management of thyroid nodules--2016 update. Endocr Pract. 2016;22(5):622-639.

9. Ferris RL, Baloch Z, Bernet $\mathrm{V}$, et al. American thyroid association statement on surgical application of molecular profiling for thyroid nodules: current impact on perioperative decision making. Thyroid. 2015;25(7):760-768.
10. Alexander EK, Kennedy GC, Baloch ZW, et al. Preoperative diagnosis of benign thyroid nodules with indeterminate cytology. The New England journal of medicine. 2012;367(8):705-715.

11. Zhang M, Lin O. Molecular testing of thyroid nodules: a review of current available tests for fine-needle aspiration specimens. Arch Pathol Lab Med. 2016;140(12):1338-1344.

12. Nikiforov YE, Carty SE, Chiosea SI, et al. Highly accurate diagnosis of cancer in thyroid nodules with follicular neoplasm/suspicious for a follicular neoplasm cytology by ThyroSeq v2 next-generation sequencing assay. Cancer. 2014;120(23):3627-3634.

13. Valderrabano P, Khazai L, Leon ME, et al. Evaluation of ThyroSeq v2 performance in thyroid nodules with indeterminate cytology. Endocr Relat Cancer. 2017;24(3):127-136. 